\title{
IMPACTO AMBIENTAL SUSTENTÁVEL COM A IMPLANTAÇÃO DO PROTOCOLO DO FLUXO DIGITAL NA REDUÇÃO DOS RESÍDUOS NA CLÍNICA ODONTOLÓGICA
}

\author{
SUSTAINABLE ENVIRONMENTAL IMPACT WITH THE IMPLEMENTATION OF THE \\ DIGITAL FLOW PROTOCOL TO REDUCE WASTE AT THE DENTAL CLINIC
}

\author{
Denise Campos Amaral', Rodrigo Augusto Soto Verri², Geovanna Rafael Martins ${ }^{3}$, Larissa Caldeira Alves Mendes ${ }^{3}$, Mayara \\ Barbosa Viandelli Mundim ${ }^{4}$ Diogo Rodrigues Cruvinel $^{5}$, Carolina Cintra Gomes ${ }^{6}$ \\ 1 - Especialista em Odontopediatria e aluna do Programa de Pós-Graduação em Odontologia, Centro Universitário de Anápolis - \\ UniEVANGÉLICA, Anápolis, Goiás, Brasil. \\ 2 - Especialista em Dentística e aluno do Programa de Pós-Graduação em Odontologia, Centro Universitário de Anápolis - UniEVANGÉLICA, \\ Anápolis, Goiás, Brasil. \\ 3 - Graduação em Odontologia, Centro Universitário de Anápolis - UniEVANGÉLICA, Anápolis, Goiás, Brasil. \\ 4 - Doutora em Clínica Odontológica, Área de Diagnóstico, professora do Curso de Odontologia e Programa de Pós-Graduação do Centro \\ Universitário de Anápolis - UniEVANGÉLICA, Anápolis, Goiás, Brasil. \\ 5 - Doutor em Reabilitação Oral, Área de Prótese Dentária, professor do Curso de Odontologia e Programa de Pós-Graduação do Centro \\ Universitário de Anápolis - UniEVANGÉLICA, Anápolis, Goiás, Brasil. \\ 6 - Doutora em Radiologia Odontológica e Imaginologia, Área de Diagnóstico, professora do Curso de Odontologia e Programa de Pós-Graduação \\ do Centro Universitário de Anápolis - UniEVANGÉLICA, Anápolis, Goiás, Brasil.
}

\section{RESUMO}

Objetivo: O objetivo neste estudo foi contabilizar a quantidade de resíduos, provenientes dos filmes radiográficos convencionais, que deixou de ser produzido com a implantação do sistema de imagem digital no Centro de Diagnóstico por Imagem da UniEVANGÉLICA (CDI). Material e Métodos: Foram contabilizados 2554 exames intrabucais realizados durante o ano letivo de 2017. Cada componente do filme radiográfico intrabucal (película de chumbo, papel preto e envelope plástico) foi pesado individualmente e comparados com o peso do plástico usado para isolar o sensor do tipo placa de fósforo durante a tomada radiográfica. Esses dados possibilitaram o cálculo da quantidade de resíduos gerados a partir desses exames. Resultados: Após teste de porcentagem simples observou-se uma redução de $97 \%$ em peso total de resíduos gerados com o uso da Radiologia Digital. Conclusão: A partir dos resultados percebe-se uma redução significativa na quantidade de resíduos gerados pela radiologia após a implantação do sistema de imagem digital no CDI. Atualmente o CDI oferece aos pacientes exames de maior qualidade se adequando ao ambiente de Sistema de Comunicação e Arquivamento de Imagens (PACS) além de apresentar um impacto ambiental positivo com a redução de resíduos gerados.

Palavras-chaves: Resíduos de Serviços de Saúde; Filme radiográfico; Diagnóstico por imagem; Meio ambiente; Indicadores de Desenvolvimento Sustentável.

\section{INTRODUÇÃO}

Na Odontologia, existem procedimentos que geram resíduos que podem ser nocivos à saúde de pacientes e profissionais submetidos a um contato direto ou indireto. Dentre os efluentes gerados, os mais importantes são os materiais perfurocortantes e com contaminação de saliva ou sangue; restos de amálgama; lâminas de chumbo provenientes das películas radiográficas e soluções processadoras de filme radiográfico; soluções desinfetantes; medicamentos que não devem ser consumidos ${ }^{1}$.

A radiografia é uma importante ferramenta complementar utilizada para diagnósticos na área da saúde. Entretanto, além dos efluentes gerados oriundos do processamento (revelador, fixador e água de lavagem de filmes radiográficos) ocorre à produção de resíduos sólidos, os componentes do filme radiográfico (filme, película de chumbo, papel preto e envelope plástico). Estes resíduos representam problemas ambientais, pois contêm compostos orgânicos e inorgânicos, tóxicos ao meio ambiente, quando descartados inadequadamente ${ }^{2-4}$.

Contudo, o problema não é a produção de resíduos, mas o destino final ligado à viabilidade de tratamento deles. Fenômeno agravado pela falta de recursos financeiros e tecnológicos para que haja o gerenciamento desses resíduos, situação retratada, principalmente, nos países em desenvolvimento ${ }^{5}$.

Em alguns casos, os resíduos radiológicos podem ser reciclados (como exames radiográficos descartados, dos quais se pode 
recuperar a prata e a base de poliéster), reutilizados (como as caixas de filmes extrabucais, as quais podem ser empregadas para armazenar radiografias), ou devem ser tratados antes de serem desprezados no meio ambiente ${ }^{6}$.

A radiografia digital representa um grande avanço tecnológico, permitindo recursos indisponíveis nas técnicas convencionais, no entanto, as bases técnicas, assim como a fonte de energia continuam inalteradas ${ }^{7}$. O método de obtenção é feito substituindo o filme e o processamento convencional por receptores ou sensores e um computador ${ }^{8}$.

Dentre as vantagens da radiografia digital estão: a possibilidade de manipulação da imagem para que as informações nela contidas tornem-se mais facilmente detectáveis, a facilidade para mensurações e cálculos a respeito de dimensões e variações de densidade, a eliminação da necessidade de câmara escura e do processamento químico, a redução de até $80 \%$ da dose de radiação utilizada para obtenção da imagem e, a celeridade dos processos de arquivamento, comparações, obtenção de cópias e transmissão à distância ${ }^{9}$,sendo estas as principais justificativas no desenvolvimento e lançamento comercial dos diversos sistemas de radiografia digital no mercado ${ }^{10}$.

Diante da preocupação com o meio ambiente, e das novas tecnologias utilizadas na Radiologia Odontológica, esse estudo tem como objetivo avaliar a quantidade de resíduos provenientes dos filmes radiográficos convencionais que deixou de ser produzido com a implantação do sistema de imagem digital no Centro de Diagnóstico por Imagem da UniEVANGÉLICA.

\section{MATERIAL E MÉTODO}

Essa pesquisa quantitativa, observacional de caráter transversal, foi realizada no Centro de Diagnóstico por Imagem (CDI) do Centro Universitário de Anápolis (UniEVANGÉLICA).

Foi realizado um levantamento sobre a quantidade de radiografias intrabucais realizadas durante o ano letivo de 2017. Foram contabilizados 2554 exames intrabucais realizados durante o referido período. O número amostral foi obtido por meio dos relatórios mensais de exames intrabucais realizados no CDI, contabilizando a quantidade de exames realizados no atendimento à pacientes no Curso de Odontologia durante um período de nove meses. Esses dados possibilitaram o cálculo da quantidade de resíduos gerados a partir desses exames intrabucais convencionais, durante esse período.

A coleta dos dados ocorreu em local reservado, onde apenas os envolvidos na pesquisa tiveram acesso às informações do relatório de atendimento do CDI, garantindo a privacidade e confidencialidade dos dados acessados. Após a coleta, os valores obtidos foram anexados em uma tabela do software Microsoft Office Excel ${ }^{\circledR}$ (Versão: 16.0, 2019).

Para pesagem de cada componente do filme radiográfico convencional intrabucal, foi utilizada uma balança semianalítica (GEHAKA BK660, São Paulo - SP) (Figura 1-5). Com a mesma balança foi realizada a pesagem da embalagem plástica descartável utilizada como barreira de proteção envolvendo o sensor radiográfico (Figura 6).

O peso unitário de cada componente do filme radiográfico e do plástico utilizado como barreira de proteção para o sensor radiográfico foi multiplicado pela quantidade total de radiografias intrabucais realizadas durante o ano letivo. Os valores dos pesos de cada componente do filme radiográfico foram somados totalizando a quantidade final de resíduos gerados em gramas com o uso da radiologia convencional. $\mathrm{O}$ peso unitário do plástico utilizado como barreira de proteção para o sensor radiográfico multiplicado pela quantidade total de radiografias intrabucais realizadas durante o ano letivo totalizou a quantidade final de resíduos gerados em gramas com o uso da radiologia digital.

Os resultados foram tabulados e uma porcentagem simples aplicada comparando o peso total dos resíduos gerados com uso da radiologia convencional versus a radiologia digital.

\section{RESULTADOS}

Os resultados apresentaram a produção de 5.207,6g de resíduos gerados com a utilização de filmes radiográficos, comparada a $150,686 \mathrm{~g}$ com a utilização de sensores radiográficos. Esses resultados podem ser observados de forma discriminada nas tabelas 1 e 2 .

Após teste de porcentagem simples observou-se uma redução de $97,106 \%$ em peso de resíduos gerados com o uso da Radiologia Digital.

\section{DISCUSSÃO}

Com o advento da radiologia digital houve a substituição do filme e do processamento convencional por sensores radiográficos e computadores ${ }^{8}$.

O sistema digital dispensa apresenta vantagens em relação ao sistema convencional, com a eliminação de resíduos, devido à dispensa do processamento químico e o uso de sensores radiográficos que eliminam o descarte do invólucro do filme convencional. Essa vantagem foi mensurada nesse estudo em que se comparou o peso dos resíduos gerados entre o sistema convencional e o sistema digital. Enquanto, para realizar 2554 radiografias intrabucais no sistema convencional gerava-se 5.207,606g de resíduos; pelo sistema digital a mesma quantidade de radiografias pôde ser realizada gerando 150,686g de resíduos de material reciclável.

Comparando a quantidade de resíduos gerados pelos sistemas convencional e digital durante o ano letivo de 2017, após teste de porcentagem simples observou-se uma redução de 97,106\% em peso de resíduos gerados com o uso do sistema digital.

Tabela 1 - Relação dos resíduos gerados com a Radiologia Convencional, peso individual e total durante um ano letivo

\begin{tabular}{llll}
\hline Componentes & Quantidade/ano & Peso unitário $(\mathrm{g})$ & Peso total $(\mathrm{g})$ \\
\hline Filme & 2554 & 0,347 & 886,238 \\
\hline Película de chumbo & 2554 & 0,577 & 1473,658 \\
\hline Papel preto & 2554 & 0,274 & 699,796 \\
\hline Envelope plástico & 2554 & 0,841 & 2147,914 \\
\hline
\end{tabular}

Tabela 2 - Relação dos resíduos gerados com a Radiologia Digital, peso individual e total durante um ano letivo

\begin{tabular}{llll}
\hline Componente & Quantidade/ano & Peso unitário $(\mathrm{g})$ & Peso total $(\mathrm{g})$ \\
\hline $\begin{array}{l}\text { Plástico } \\
\text { (Barreira de proteção) }\end{array}$ & 2554 & 0,059 & 150,686 \\
\hline
\end{tabular}




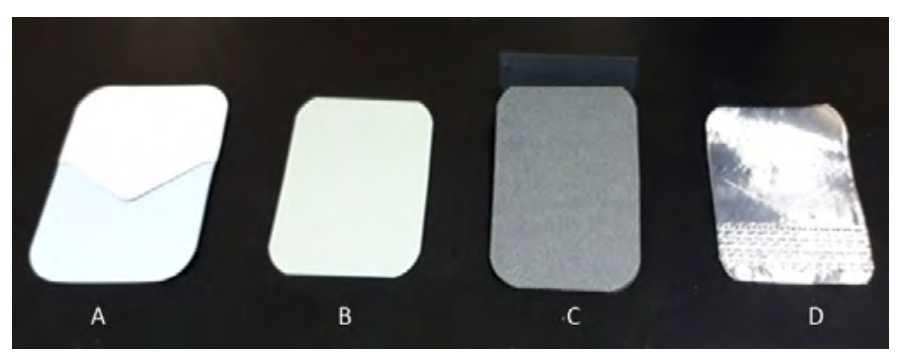

Figura 1 - Componentes do filme radiográfico: envelope plástico (A), Filme (B), papel preto (C) e película de chumbo (D).

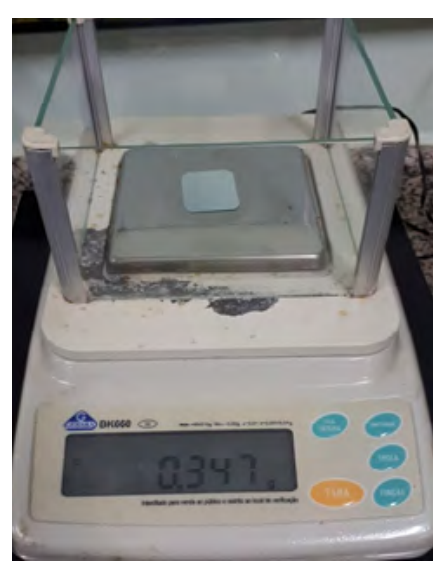

Figura 2 - Pesagem do filme

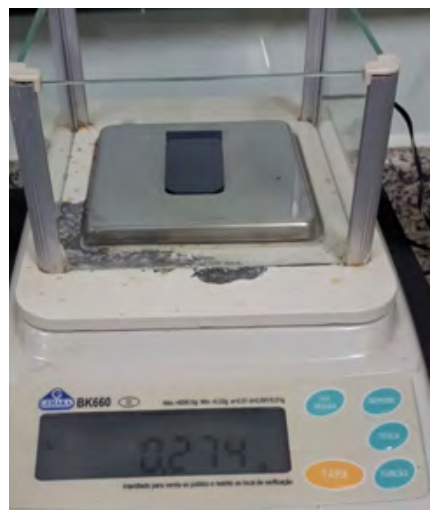

Figura 4 - Pesagem do papel preto

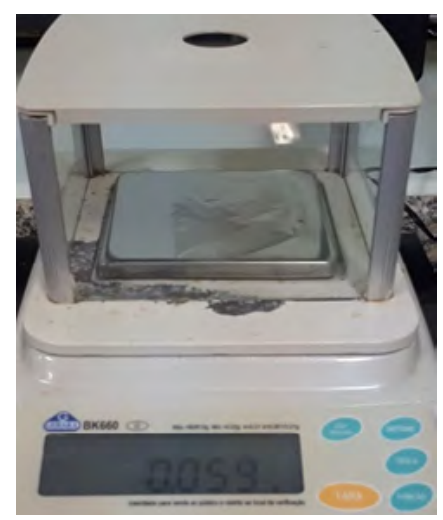

Figura 5 - Pesagem do plástico utilizado como barreira de proteção para o sensor radiográfico

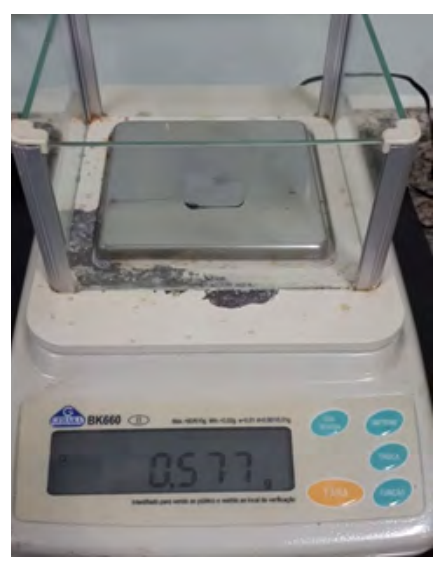

Figura 3 - Pesagem da película de chumbo

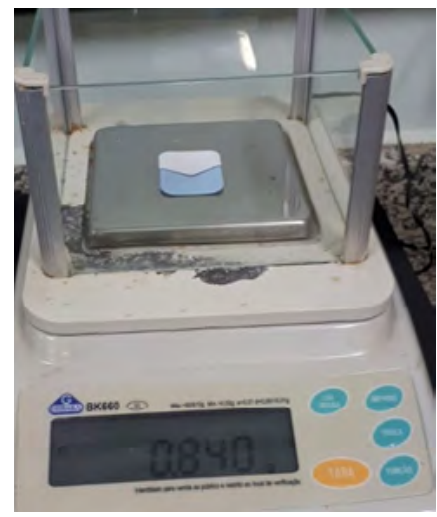

Figura 5 - Pesagem do envelope plástico
Entretanto, mesmo com todas as vantagens desse sistema, em alguns serviços o sistema convencional ainda é utilizado. A não implementação unânime do sistema de radiologia digital está diretamente relacionada ao alto custo do mesmo. Todavia essa substituição está ocorrendo de forma gradual, pois as vantagens do sistema digital apresentam benefícios diretos e indiretos à saúde do ser humano, inviabilizando a manutenção do sistema convencional. De forma indireta ao ser humano, o meio ambiente é contaminado por soluções para processamento radiográfico (revelador e fixador) que contém metais pesados nocivos ao meio ambiente, além da película de chumbo e o papel preto ${ }^{2}$, elementos estes que não podem ser descartados em lixo comum ${ }^{11}$.

Em uma diretriz a respeito das recomendações sobre intervenções digitais para o fortalecimento do sistema de saúde, a Organização Mundial da Saúde recomendou o uso da modalidade digital para melhorar a saúde das pessoas ${ }^{12}$. Na Itália, uma estratégia para promover o crescimento digital está sendo desenvolvida e, recentemente, o governo italiano definiu um documento de política estratégica e econômica para um plano de três anos (2019-2021) para Tecnologia da Informação na Administração Pública ${ }^{13}$, que também definiu as ações de intervenção dedicadas à saúde digital e as principais soluções para a evolução do sistema de informação da administração pública, visando melhorar serviços de saúde, limitando desperdícios e ineficiências, melhorando a relação custo-qualidade dos serviços de saúde e a redução das diferenças entre territórios ${ }^{14}$.

Portanto, a tecnologia digital possibilitou vários benefícios na área da saúde e para o meio ambiente. Além da redução de resíduos analisada nesse estudo, com a substituição do processamento radiográfico convencional pelo processamento digital não são gerados efluentes radiográficos provenientes dos agentes químicos do processamento convencional, evitando o lançamento desses resíduos no meio ambiente ${ }^{15}$.

No Brasil, a substituição da tecnologia convencional pela digital tem ocorrido de forma gradual, principalmente nos serviços de radiodiagnóstico; no entanto, por envolver custos elevados, nem todos os estabelecimentos têm os recursos para aquisição dos equipamentos necessários ${ }^{15}$.

\section{CONCLUSÕES}

A partir desse estudo certificamos a redução significativa na quantidade de resíduos provenientes dos filmes radiográficos convencionais, que deixou de ser produzido com a implementação do sistema de imagem digital no Centro de Diagnóstico por Imagem da UniEVANGÉLICA.

Com essa pesquisa buscamos a conscientização da importância de atualizar-se e utilizar novas tecnologias, a fim de proteger o meio ambiente contra a poluição proveniente dos resíduos radiográficos. Atualmente o CDI oferece aos pacientes exames de maior qualidade se adequando ao ambiente de Sistema de Comunicação e Arquivamento de Imagens (PACS) além de apresentar um impacto ambiental positivo com a redução de resíduos gerados.

\section{REFERÊNCIAS}

01. Gusmão M. A importância do plano de gerenciamento de resíduos odontológicos nas organizações militares de saúde do exército brasileiro [Trabalho de Conclusão de Curso]. Rio de Janeiro: Escola de Saúde de Exército; 2009. 
02. Ues $\mathrm{K}$ et al. Uso de processos avançados de oxidação na degradação dos resíduos de revelador e fixador de raio-x. Anais do XVI Encontro de Química da Região Sul; 13-15 nov 2008; Blumenau (SC); 2008.

03. Guedes DFCS, Reginaldo Santana da Veiga, Márcia Andréia Mesquita Silva da Sousa Neto, Manoel Damião de Pécora, Jesus Djalma. O papel preto da película radiográfica é um alto risco para o meio ambiente. Rev Assoc Paul Cir Dent. 2009;63:191 - 4.

04. Freitas A, Rosa JE, Souza IF. Radiologia odontológica. 6 ed. São Paulo: Artes Médicas; 2004.

05. Morais L. Avaliação do plano de gerenciamento de resíduos de serviços de saúde da área odontológica [Dissertação de Mestrado]. Anápolis: Centro Universitário de Anápolis; 2011.

06. Molina $\mathrm{AB}$, et al. A radiologia odontológica e o meio ambiente. Rev. Odontol. 2014,26(1):61-70.

07. Botelho TL, Mendonça EF, Cardoso LLM. Contribuição na Radiografia digital na clínica odontológica. Rev. Robrac. 2003; 12(33): 55-9.

08. Tavano O, Silva MAGS. A radiografia digital na odontologia. Rev Fac Odontol. 1999,1(1):52-5.

09. Haiter Neto F, et al. Estágio atual da radiografia digital. Rev. ABRO. Brasília 2000; 1(3): 1-6.
10. Attaelmanan A, Borg E, Grondahl HG. Digitisation and display of intra-oral films. Dentomaxillofac Radiol. 2000;29(2):97-102.

11. Brasil. Ministério do Meio Ambiente. Conselho Nacional do Meio Ambiente (CONAMA). Resolução n³58, de 29 de abril de 2005. Dispõe sobre o tratamento e a disposição final dos resíduos dos serviços de saúde e dá outras providências. Diário Oficial da União, Brasília, DF, 04 maio 2005: 63-65.

12. Organização Mundial de Saúde. Diretriz da OMS: Recomendações sobre intervenções digitais para o fortalecimento do sistema de saúde. Genebra: OMS; 2019.

13. Agenzia per l'Italia Digitale (AGID). Piano Triennale Sanità digitale: https://www.agid.gov.it/it/piattaforme/sanita-digitale. Accessed 2 Feb 2020.

14. Cristofaro M, Piselli P, Pianura E, et al. Patient Access to an Online Portal for Outpatient Radiological Images and Reports: Two Years' Experience. J Digit Imaging.2020;10.

15. Grigoletto JC, Segura-Muñoz SI, Barbosa-Junior F, Sanches SM, Takayanagui AM. Silver discharged in effluents from imageprocessing services: a risk to human and environmental health. Biol Trace Elem Res. 2011;144(1-3):316-326. doi:10.1007/s12011-011-9096-8.

\section{ABSTRACT}

Objective: The goal of this study was to account for the amount of radiographic film residues that were no longer produced with the implementation of a digital imaging system at the UniEVANGÉLICA Diagnostic Imaging Center (CDI). Materials and Methods: 2554 intraoral examinations were carried out during the academic year of 2017. Each component of the intraoral radiographic film (lead film, black paper and plastic envelope) was weighed and compared to the weight of the plastic used to isolate the sensor and to the phosphor plate during a radiographic intake. This data enable us to calculate the amount of waste generated from these tests. Results: After the simple percentage test, a 97\% reduction in the total weight of waste generated with the use of Digital Radiology is observed. Conclusion: Based on the results, a significant reduction in the amount of waste generated by radiology can be seen after the implementation of the digital imaging system at CDI. Currently, CDI offers patients higher quality tests, appropriate to the environment of the Image Archiving and Communication System (PACS), in addition to offering a positive environmental impact with a reduction in the waste generated.

Keywords: Medical Waste; X-Ray Film; Diagnostic Imaging; Enviroment; Sustainable Development Indicators.

\section{AUTOR PARA CORRESPONDÊNCIA}

Carolina Cintra Gomes

Endereço: Av. Universitária Km 3,5 Cidade Universitária

- Anápolis/GO.

CEP: 75083-515

Telefone: +55 (62) 982712244

e-mail: carolcintrag@hotmail.com 